\title{
Regulatory conservation of protein coding and microRNA genes in vertebrates: lessons from the opossum genome Shaun Mahony ${ }^{*}$, David L Corcoran ${ }^{\dagger}$, Eleanor Feingold ${ }^{\dagger \ddagger}$ and Panayiotis V Benos ${ }^{* \dagger \S}$
}

\begin{abstract}
Addresses: *Department of Computational Biology, School of Medicine, University of Pittsburgh, Fifth Avenue, Pittsburgh, PA 15260, USA. ${ }^{\dagger}$ Department of Human Genetics, Graduate School of Public Health, University of Pittsburgh, DeSoto Street, Pittsburgh, PA 15261, USA. ${ }^{\ddagger}$ Department of Biostatistics, Graduate School of Public Health, University of Pittsburgh, DeSoto Street, Pittsburgh, PA 15261, USA. \$University of Pittsburgh Cancer Institute, School of Medicine, University of Pittsburgh, Centre Avenue, Pittsburgh, PA 15232, USA.
\end{abstract}

Correspondence: Panayiotis V Benos. Email: benos@pitt.edu

Published: 16 May 2007

Genome Biology 2007, 8:R84 (doi:10.1186/gb-2007-8-5-r84)

The electronic version of this article is the complete one and can be found online at http://genomebiology.com/2007/8/5/R84
Received: 6 November 2006

Revised: 29 January 2007

Accepted: 16 May 2007

(c) 2007 Mahony et al.; licensee BioMed Central Ltd.

This is an open access article distributed under the terms of the Creative Commons Attribution License (http://creativecommons.org/licenses/by/2.0), which permits unrestricted use, distribution, and reproduction in any medium, provided the original work is properly cited.

\begin{abstract}
Background: Being the first noneutherian mammal sequenced, Monodelphis domestica (opossum) offers great potential for enhancing our understanding of the evolutionary processes that take place in mammals. This study focuses on the evolutionary relationships between conservation of noncoding sequences, cis-regulatory elements, and biologic functions of regulated genes in opossum and eight vertebrate species.

Results: Analysis of 145 intergenic microRNA and all protein coding genes revealed that the upstream sequences of the former are up to twice as conserved as the latter among mammals, except in the first 500 base pairs, where the conservation is similar. Comparison of promoter conservation in $5 / 3$ protein coding genes and related transcription factor binding sites (TFBSs) showed that $41 \%$ of the known human TFBSs are located in the $6.7 \%$ of promoter regions that are conserved between human and opossum. Some core biologic processes exhibited significantly fewer conserved TFBSs in human-opossum comparisons, suggesting greater functional divergence. A new measure of efficiency in multigenome phylogenetic footprinting (base regulatory potential rate [BRPR]) shows that including human-opossum conservation increases specificity in finding human TFBSs.

Conclusion: Opossum facilitates better estimation of promoter conservation and TFBS turnover among mammals. The fact that substantial TFBS numbers are located in a small proportion of the human-opossum conserved sequences emphasizes the importance of marsupial genomes for phylogenetic footprinting-based motif discovery strategies. The BRPR measure is expected to help select genome combinations for optimal performance of these algorithms. Finally, although the etiology of the microRNA upstream increased conservation remains unknown, it is expected to have strong implications for our understanding of regulation of their expression.
\end{abstract}




\section{Background}

One of the prime motivating factors driving the sequencing of vertebrate genomes is the expectation that the role played by the functional regions of the human genome may be discerned by finding molecular level commonalities with and differences from other animals. This is especially true of the newly sequenced opossum (Monodelphis domestica), which is the first completed marsupial genome. Being the first noneutherian mammal sequenced, the opossum helps to clarify which sequence changes occurred before and after the divergence of mammalian ancestors from other vertebrates [1], and has already provided new insight into the evolution of mammalian major histocompatibility complex genes [2]. It is also hoped that the opossum genome may yield insights into how gene regulation has evolved in vertebrates.

In protein coding genes, gene regulation is primarily controlled by short DNA sequences in the vicinity of the gene's transcription start sites (TSSs), which are targets for transcription factor proteins. A high degree of evolutionary conservation of these promoter regions can be attributed to functional cisregulatory elements. The increased conservation in the biologically more important parts of the promoter region has been explored by various phylogenetic footprinting algorithms, such as PhyloGibbs [3], ConSite [4], rVista [5], and FOOTER [6], to improve the prediction of transcription factor binding sites (TFBSs) in vertebrate genomes. Phylogenetic footprinting is a comparative genomics approach that exploits cross-species sequence conservation in order to predict regulatory genomic elements. In the absence of evolutionary information, TFBSs can be evaluated in terms of sequence similarity scans against frequency matrices derived from alignments of known binding sites for a given transcription factor [7]. However, the typical short length of TFBSs (5 to 20 base pairs [bp]) and their inherent level of sequence degeneracy makes them notoriously difficult to predict with any degree of specificity using similarity searches alone [8]. Phylogenetic footprinting provides a way to reduce the sequence search space to regions that are conserved (and therefore more likely to contain functional elements), thereby improving the specificity of TFBS prediction.

In order to improve the performance of phylogenetic footprinting algorithms, the evolutionary aspects of the promoter regions and the TFBSs residing in them must be investigated. Evolutionary distance is an important factor in the effectiveness of phylogenetic footprinting techniques. For example, the divergence between chimpanzee and human is generally insufficient to reduce the sequence search space in any meaningful way; conversely, the divergence between Drosophila and human can be too large for any regulatory sequence conservation to be detected. Recently, the maximum sensitivity of phylogenetic footprinting techniques has been measured via estimations of the rate of TFBS 'turnover' between human and rodent genomes [9-13]. We consider that a TFBS has undergone turnover if the sequence in which it resides is not conserved between the species compared. High or low TFBS turnover rates do not necessarily coincide with the rate of changes in the regulatory mechanism (for instance, replacement TFBSs can arise by chance elsewhere in the promoter region or functional TFBSs may still be present in nonconserved regions). Turnover, however, corresponds to the minimum false-negative rate for detection of TFBSs via phylogenetic footprinting, and thus it serves as a critical bound on the success of such algorithms. Human-rodent TFBS turnover has been estimated at between $28 \%$ and $40 \%$ [9-13], suggesting that TFBSs are among the most malleable functional elements in the genomic landscape. However, although rodents and primates diverged relatively recently (approximately 90 million years ago [14]), the shorter generational time of rodents has placed a large degree of dissimilarity between the two clades, as is evident in the human-dog comparisons [15]. Therefore, TFBS turnover rates will have to be estimated in other mammals before a clearer picture of the selective pressure on mammalian TFBSs can emerge.

Another major mechanism for control of gene expression is provided by microRNA (miRNA) genes. miRNAs are small (22 to $61 \mathrm{bp}$ long), noncoding RNAs that downregulate their target genes via base complementarity to their mRNA molecules $[16,17]$. Each miRNA can target multiple genes and each gene can be targeted by multiple miRNAs [18-21]. In vertebrates, their expression is tissue specific [22] and has been shown to play an important role during development [23-25]. Although some miRNAs are found in the introns of coding genes and therefore are probably regulated by the promoters of the genes in which they reside [26], others are located in the intergenic parts of the genome. Little is known about the transcriptional regulation of these intergenic miRNAs, although RNA polymerase II appears to be involved in the process [27]. This suggests that they may have active promoter regions that contain cis-regulatory elements, similar to coding genes. The following question then arises; how does the conservation in the upstream regions of the intergenic miRNA genes compare with that of the protein coding genes? In this respect, opossum and the other vertebrate species provide a broad range of evolutionary distances in which this issue may be addressed.

In this report we present our findings regarding promoter conservation of all protein coding genes and upstream sequence conservation of intergenic miRNA genes in eight vertebrate genomes as compared with human. To our knowledge, this is the first time that such a comprehensive study has been conducted on potential regulatory regions of both protein coding and miRNA genes in vertebrates. Also, because the opossum genome is placed at an evolutionary midpoint relative to eutherian mammals and nonmammalian vertebrates, using it as an outgroup to the existing eutherian genomes allows for the estimation of the mammalian TFBS turnover rate. Furthermore, the opossum genome provides an opportunity to assess which transcriptional signals and 


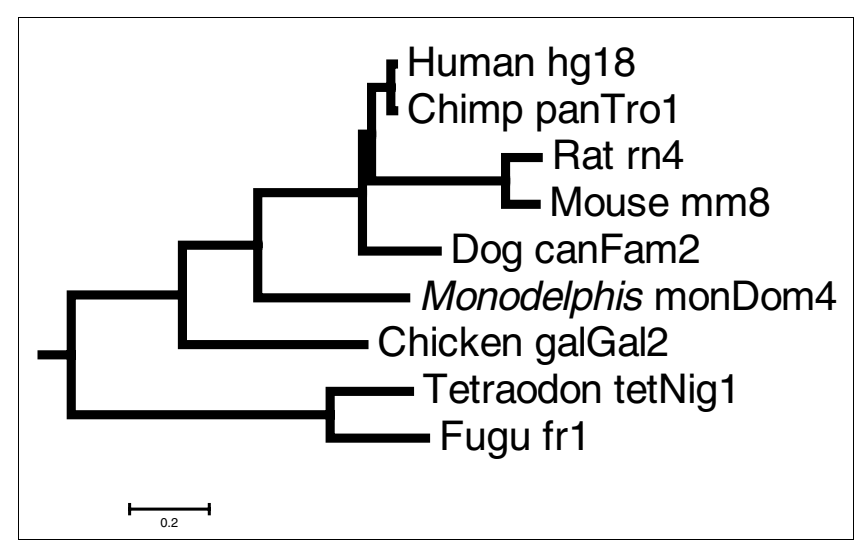

Figure I

Phylogenetic tree of the species examined in this study. This phylogenetic tree is based on the University of California, Santa Cruz (UCSC) multiple alignments. The tree was generated using phyloGif [72].

regulatory mechanisms are shared between all mammals. For these reasons, the conservation rates of the promoters of 513 human genes are also analyzed in relation to the turnover of the 1,162 TFBSs they contain. Relationships between conservation of sites and identity of the corresponding transcription factors and their Gene Ontology (GO) [28] categories are also investigated. Finally, we computationally re-evaluate the potential of phylogenetic footprinting in the light of the opossum genome and other recently sequenced vertebrates. A new statistical measure, the base regulatory potential rate (BRPR), is introduced to assess the efficiency of both pairwise and multiple species comparisons in phylogenetic footprinting strategies.

\section{Results and discussion \\ Distribution of conserved blocks in the upstream} regions of protein coding and intergenic miRNA genes Conservation of the 5 kilobases $(\mathrm{kb})$ upstream regions of all RefSeq protein coding genes as well as the known intergenic miRNA genes was calculated using the sliding window approach, as we describe in Materials and methods (below). We chose to focus solely on intergenic miRNAs because intronic miRNAs have been shown to be co-transcribed with their corresponding protein coding genes [26]. Because little is known about the transcriptional regulation of non-intronic miRNA genes, we cannot assess the possible TFBS turnover. We can, however, assess whether the miRNA upstream regions evolve at the same, slower, or faster rate than those of the protein coding genes, and whether their conservation pattern across the upstream region indicates parts of potential biologic importance. The phylogenetic tree of the species examined in this paper is plotted in Figure 1.

Table 1 presents the number of orthologous genes in each species (derived from the MULTIZ University of California,
Santa Cruz [UCSC] synteny-based alignments), the average block coverage of their upstream regions, and the average percentage identity within these conserved blocks. For the calculation of the average percentage identity, the conservation percentage of each block is multiplied by the total length of the block. In other words, the average block conservation corresponds to the number of bases that are identical in all conserved blocks of one promoter over the total length of the blocks in this promoter. The human genes were used as reference for all pair-wise comparisons. Surprisingly, we found that, with the exception of teleosts and chimp, the conservation in the upstream regions of the miRNA genes is $34 \%$ to $60 \%$ higher on average than that in the protein coding genes. This is independent of the average block identity, which remains practically the same between the two types of genes in these comparisons (Table 1). In all nonprimate mammals the average block coverage in the miRNA upstream sequences is significantly higher than that in the promoters of the protein coding genes (Wilcoxon rank-sum test: $P=6 \times 10^{-4}$ for opossum and $P=10^{-14}$ to $10^{-16}$ for rodents and $\operatorname{dog}$ ).

In order to investigate this surprising finding further, we plotted the sequence conservation as a function of the distance from the start of the corresponding genes (Figure 2). We found that in the first $500 \mathrm{bp}$ the sequence conservation of the miRNA genes is almost identical to that of the promoters of the protein coding genes ( $R$ values $>0.9$ and usually much higher; regression $t$-test: $P<10^{-19}$ ). In protein coding genes this is typically the region with the highest concentration of the known cis-regulatory elements. From all known human and mouse TFBSs in TRANSFAC [29], 69.1\% and 65.1\%, respectively, are annotated as being located in the proximal 500 bp region (data not shown). Interestingly, Lee and coworkers [27] showed that this region is sufficient to drive expression of the miR 23a 27a 24-2 intergenic miRNA gene cluster by RNA polymerase II. Could this be a coincidence? We tested this by analyzing the upstream sequence conservation of the tRNA genes in the human genome (see Materials and methods, below). It has been long established that the cis-regulatory elements of the tRNA genes are located downstream of their transcription start [30]. We found that the sequence conservation for the tRNA genes was constant throughout their $5 \mathrm{~kb}$ upstream regions (Figure 2; green dashed line).

The conservation rates in both protein coding and miRNA genes decline after the first $500 \mathrm{bp}$ and become almost constant. The difference between these two types of genes is that, in the case of miRNAs, the constant conservation rate is up to twofold higher than that in the protein coding genes for rodents, dog, opossum, and chicken. We found this difference to be statistically significant (Additional data file 1 [Supplementary Figure 2]). Similarly high conservation rates are observed in chimp for both types of genes, probably reflecting the generally high conservation rate throughout the genome. By contrast, similarly low conservation rates are observed for 
Table I

Conservation in the 5 kilobases upstream sequences in all protein coding and intergenic miRNA genes

\begin{tabular}{|c|c|c|c|c|c|c|c|}
\hline \multirow[t]{2}{*}{ Human versus } & \multicolumn{3}{|c|}{ Protein coding genes } & \multicolumn{3}{|c|}{ Intergenic miRNA genes } & \multirow{2}{*}{$\begin{array}{l}\text { Relative } \\
\text { conservation }\end{array}$} \\
\hline & $\begin{array}{l}\text { Number of } \\
\text { orthologous }\end{array}$ & Block coverage & $\begin{array}{l}\text { Average block } \\
\text { identity }\end{array}$ & $\begin{array}{l}\text { Number of } \\
\text { orthologous }\end{array}$ & Block coverage & $\begin{array}{l}\text { Average block } \\
\text { identity }\end{array}$ & \\
\hline Chimp & 23,643 & $93.03 \%$ & $98.15 \%$ & 144 & $93.46 \%$ & $98.51 \%$ & $0.46 \%$ \\
\hline Mouse* & 22,790 & $23.30 \% *$ & $73.53 \%$ & 142 & $36.17 \% *$ & $74.72 \%$ & $55.24 \%$ \\
\hline Rat* & 22,161 & $22.46 \% *$ & $73.49 \%$ & 140 & $34.95 \% *$ & $74.68 \%$ & $55.61 \%$ \\
\hline Dog* & 23,276 & $44.36 \% *$ & $75.58 \%$ & 145 & $61.72 \% *$ & $76.96 \%$ & $39.13 \%$ \\
\hline Opossum* & 17,334 & $7.28 \% *$ & $74.90 \%$ & 104 & $11.65 \% *$ & $76.08 \%$ & $60.03 \%$ \\
\hline Chicken & 8,087 & $4.55 \%$ & $74.87 \%$ & 54 & $6.08 \%$ & $76.80 \%$ & $33.63 \%$ \\
\hline Fugu & 6,257 & $4.13 \%$ & $72.17 \%$ & 47 & $2.73 \%$ & $73.65 \%$ & $-33.90 \%$ \\
\hline Tetraodon & 7,821 & $3.43 \%$ & $72.10 \%$ & 60 & $2.31 \%$ & $73.40 \%$ & $-32.65 \%$ \\
\hline
\end{tabular}

This table lists the number of genes orthologous to human genes in each of the genomes tested, the percentage of upstream sequence conservation (in $>65 \%$ block identity), and the weighted average within block identity. Relative conservation (in terms of block coverage) is also listed for the microRNA (miRNA) versus protein coding genes. *Species for which the block coverage of miRNA gene upstream regions is statistically significantly higher than that of the promoters of the protein coding genes.

the fugu fish and tetraodon. We note, however, that the higher conservation rates are statistically significant only in the (nonprimate) mammals, including opossum (Additional data file 1$)$.

It is not clear whether this increased upstream sequence conservation is a general biologic feature of the miRNA upstream regions or is an artifact of the methods used to discover miRNA genes. It is possible, for example, that the known intergenic miRNAs happen to fall in more conserved regions of the genome. This may be related to the way in which the miRNAs were originally identified (through high similarity to known miRNAs). However, it is also possible that because miRNAs are involved in highly regulated vital cell or organismal processes such as development [23-25], there is a much greater selective pressure on their regulatory regions. We investigate this further by comparing the upstream sequence conservation in the miRNA genes with that of genes identified as developmental according to GO classification (Figure 2; light blue dashed line). We find that the upstream conservation of the developmental genes in all mammals is uniformly higher than the overall average and similar to the conservation of the miRNA genes, especially in the first 2,00o bp. This is true for all species examined, although in the nonmammalian vertebrates the overall upstream sequence conservation for all types of genes is similarly low (10\% or lower after the first 500 bp; Figure 2). The fact that miRNA genes have been implicated in the regulation of various developmental processes [31] may partly explain the similar conservation rates in their upstream regions and the promoters of the developmental genes, also indicating that analogous mechanisms and ciselements may regulate the expression of the corresponding genes. The fact that opossum sequences also exhibit similar conservation patterns, as do the sequences of eutherian spe- cies, indicates that mammalian specific evolutionary constraints are in place.

In summary, the above observations are consistent with the idea that miRNAs are regulated by similar mechanisms as protein coding genes, which was also shown to be true in the few cases studied thus far [27,32]. As more miRNA genes are identified, the issue of their transcriptional mechanism will warrant further investigation.

In all of the above pair-wise comparisons, except humanchimp, the average block identity is about the same $(72 \%$ to $77 \%$; Table 1), regardless of the evolutionary distance or the type of gene (protein coding or miRNA). Because the block conservation threshold was $65 \%$, this equivalency indicates that a reduction in the number of conserved blocks rather than a uniform decrease in similarity is responsible for the observed conservation rates. Such a pattern of evolution is expected if the cis-regulatory sites are organized in clusters located in these upstream regions. Such clusters might contain regulatory elements specific to, for instance, primates only, eutherians only, and so on.

\section{Evolutionary turnover of transcription factor binding sites in vertebrates}

We now turn to the relationship between promoter conservation of the protein coding genes and the turnover of the cisregulatory elements located in them. Table 2 presents the percentage of known human TFBSs that reside in conserved blocks for each pair of genomes tested. The number of such detectable TFBSs in each species differs depending on the number of orthologous genes identified in that species. We note that our analysis focuses on the TFBSs that are located immediately upstream of the protein coding genes (up to 5 $\mathrm{kb}$ ). This bias is imposed by the available data. It will be inter- 


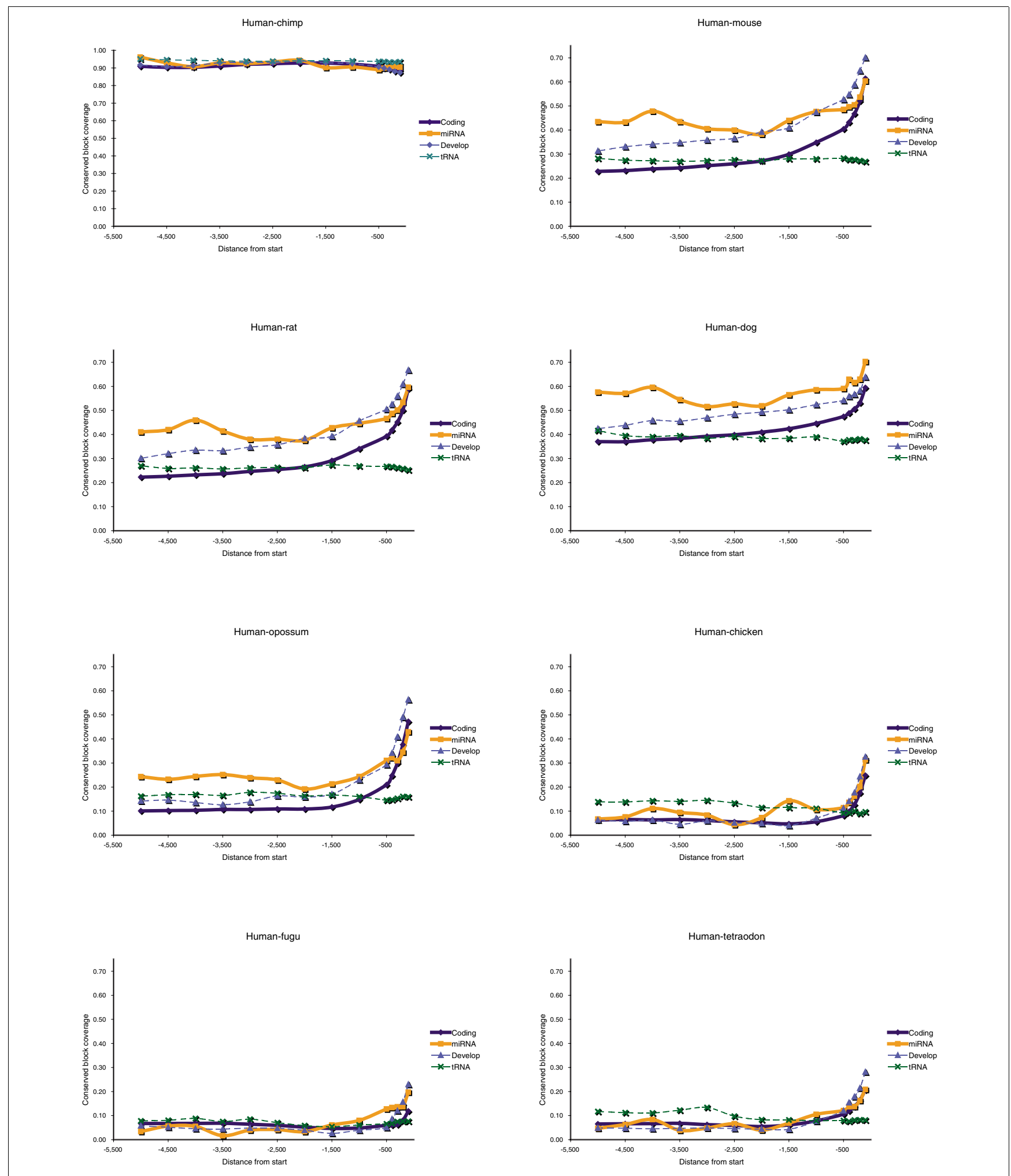

Figure 2

Upstream sequence conservation of protein coding versus miRNA genes. Comparison of 5-kilobase upstream sequence conservation between human and various organisms, relative to the transcription start site (TSS; protein-coding, solid blue line) and gene start (intergenic microRNA [miRNA] genes, orange line). The conservation of developmental genes (light blue dotted line) and tRNA genes (green dotted line) are also plotted for comparison purposes. For the plot 100 base pair (bp) intervals were used for the first 500 bp and 500 bp intervals thereafter. 


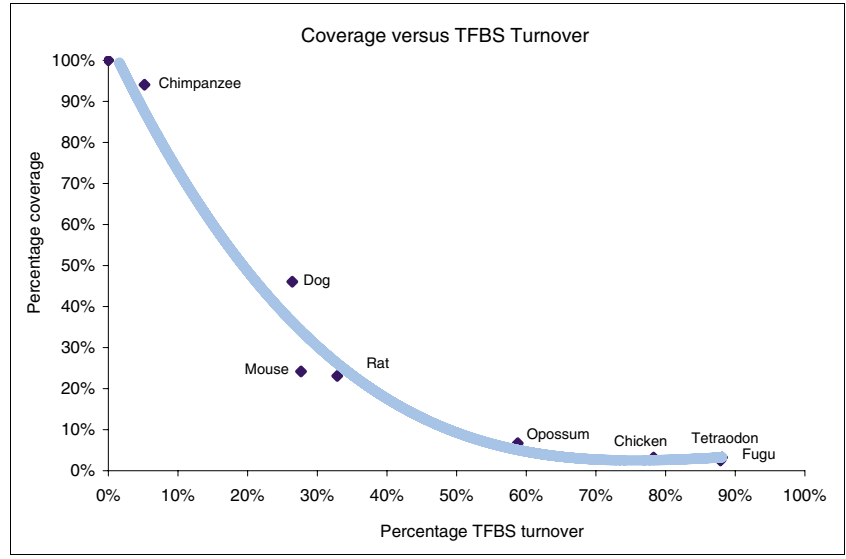

Figure 3

Conserved block coverage of the 5 kilobases upstream regions versus TFBS turnover rates. A third-order polynomial trendline is fitted for illustration. TFBS, transcription factor binding site.

esting to see how our results compare with the evolution of DNA regulatory regions in other parts of the genome.

Although we confirm previously estimated rate of humanmouse TFBS turnover [9-13], it is particularly interesting that $27 \%$ or more of the known human TFBSs are not located in blocks conserved in mammals more distant than rodents (Table 2). This does not necessarily mean that the mechanisms of gene regulation have changed accordingly. Functionally equivalent TFBSs are not always located in conserved blocks, as demonstrated in a recent comparison of gene regulation in human and zebrafish RET genes [33]. Similarly, individual TFBSs that are not conserved between two species may have been functionally replaced by other sites for the same transcription factor in one of the species [34]. The finding that only about $41 \%$ of TFBSs are located in conserved human-opossum blocks is nevertheless surprising, because it points to the relative ease with which individual mammalian TFBSs may be deleted, replaced, or added.

As expected, TFBS turnover increases with decreasing percentage conservation coverage of the upstream regions. Figure 3 shows that opossum has low block conservation similar to that in the nonmammal vertebrate species, but it retains almost twice as many sites as chicken, which is the evolutionarily closest nonmammal. This gives a first qualitative assessment for the potential importance of the opossum genome for identification of TFBSs in phylogenetic footprinting approaches. In general, outside mammalian genomes, the percentage of the detected TFBSs is reduced with increasing evolutionary distance, although the percentage $5 \mathrm{~kb}$ upstream coverage remains constant.

Table 2 also presents the average identity within the conserved TFBSs. With the exception of human-chimp comparisons, the average identity within sites is substantially higher than the average identity in the conserved blocks and rela- tively constant in all genome comparisons. We found no linear correlation between the block coverage rate and the average block identity in these comparisons $(R=0.48)$. This finding supports the idea that individual TFBSs are under greater selective pressure than are the wider conserved blocks in mammalian genomes (Wilcoxon test: $P=0.01$ ).

Finally, Table 2 presents the BRPR values for each pair of genomes (see Materials and methods, below). BRPR is the likelihood ratio of the posterior probability of a base being regulatory (part of a regulatory site), given that it is in a conserved region, over the a priori probability of being regulatory. In other words, BRPR shows how much we can improve our belief that a base (or a conserved region) is regulatory if we only focus on the conserved blocks between two or more species. One of the most surprising aspects of this study is that, on average, a relatively large percentage of TFBSs (41\%) is located in only the $6.72 \%$ of the $5 \mathrm{~kb}$ promoter regions that are conserved between human and opossum. This gives human-opossum comparisons the second highest BRPR value among the tested pair-wise comparisons, and makes the use of opossum almost twice as effective for finding regulatory elements as the more typically used human-mouse alignments (BRPR 5.647 versus 2.887, respectively). Another interesting finding is that, because of the extensive conservation between human and dog genomes, the human-dog comparisons are not as effective as human-mouse for phylogenybased motif discovery (Table 2). The maximum BRPR value occurs for human-chicken comparisons (BRPR 6.184). However, this value is very close to the opossum BRPR value and, given that only $22 \%$ of known TFBSs can be detected as conserved between human and chicken (as opposed to $41 \%$ in human-opossum), we suggest that human-opossum comparisons are more effective overall than human-chicken comparisons.

Phylogenetic footprinting becomes less effective in humanfugu and human-tetraodon comparisons (Table 2). The Afrotherian (elephant and tenrec) or Xenarthran (armadillo) genomes that are currently undergoing low-coverage sequencing, as well as the genomes of more distant vertebrates, do not appear to offer any improvement in pair-wise phylogenetic footprinting effectiveness (all are less effective than using the mouse genome; unpublished data). However, they may offer improvement in specificity in multispecies regulatory conservation scans.

\section{Phylogenetic footprinting with multispecies alignments}

Thus far, the TFBS turnover rates and BRPR values were used in pair-wise comparisons in order to assess the relative effectiveness of discovering TFBSs via evolutionary conservation. Given the availability of multiple vertebrate genomes, it is naturally expected that combining conservation information from multiple sources will increase the accuracy of phylogenetic footprinting. The following question then arises; which 
Table 2

Promoter and site conservation between human and eight vertebrate species

\begin{tabular}{|c|c|c|c|c|c|c|c|}
\hline \multirow[t]{2}{*}{ Human versus } & \multicolumn{3}{|c|}{ Promoters } & \multicolumn{3}{|c|}{ Sites } & \multirow[t]{2}{*}{ BRPR } \\
\hline & $\begin{array}{l}\text { Number of } \\
\text { orthologous } \\
\text { genes }\end{array}$ & Block coverage & $\begin{array}{l}\text { Block } \\
\text { nucleotide } \\
\text { identity }\end{array}$ & $\begin{array}{l}\text { Number of } \\
\text { detectable sites }\end{array}$ & $\%$ detected & $\begin{array}{l}\text { Site nucleotide } \\
\text { identity }\end{array}$ & \\
\hline Chimp & 512 & $94.06 \%$ & $98.27 \%$ & $\mathrm{I}, 157$ & $94.81 \%$ & $98.74 \%$ & 1.009 \\
\hline Mouse & 506 & $24.20 \%$ & $73.39 \%$ & 1,146 & $72.34 \%$ & $82.91 \%$ & 2.887 \\
\hline Rat & 496 & $23.09 \%$ & $73.21 \%$ & 1,129 & $67.14 \%$ & $83.00 \%$ & 2.757 \\
\hline Dog & 507 & $46.05 \%$ & $75.37 \%$ & $1,15 \mid$ & $73.59 \%$ & $84.77 \%$ & 1.535 \\
\hline Opossum & 389 & $6.72 \%$ & $74.63 \%$ & 912 & $41.23 \%$ & $83.93 \%$ & 5.647 \\
\hline Chicken & 189 & $3.21 \%$ & $74.43 \%$ & 451 & $21.73 \%$ & $85.06 \%$ & 6.184 \\
\hline Fugu & 127 & $3.25 \%$ & $72.87 \%$ & 286 & $11.89 \%$ & $83.98 \%$ & 3.331 \\
\hline Tetraodon & 166 & $2.50 \%$ & $73.09 \%$ & 363 & $12.12 \%$ & $80.95 \%$ & 4.227 \\
\hline
\end{tabular}

Analysis of I,162 known human transcription factor binding sites (TFBSs) associated with the promoters of 513 human genes between human and eight vertebrate species. The number of genes orthologous to human genes in each species, their conservation block coverage, and their average block identity are presented; also, the number of TFBSs associated with these orthologous genes in each species, the percentage of sites located in conserved regions between species, and the average nucleotide identity within TFBSs are reported. The base regulatory potential rate (BRPR) statistic is calculated from these data for each pair of genomes (see text). Block coverage is the percentage of the upstream region that is covered by conserved blocks (>50 base pairs with $>65 \%$ identity); the block nucleotide identity is the percentage of nucleotides in all conserved blocks that are identical to the human sequence; and site nucleotide identity the percentage nucleotides in all detected TFBSs that are identical to the human sequence.

genome combinations offer greater specificity? To address this, we evaluate all possible combinations of tested genomes (256 combinations). In the following, $P(C)$ and $P(C \mid R)$ are the prior and posterior probability, respectively, that a base is conserved, given that the base is part of a regulatory site. For consistency, both $P(C)$ and $P(C \mid R)$ are calculated over all

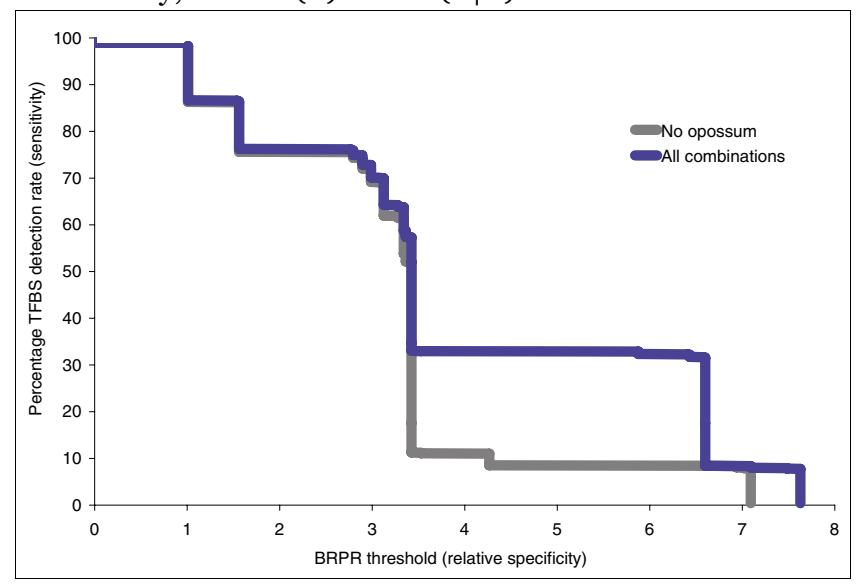

\section{Figure 4}

Association between BRPR scores and detectable sites. For each given percent of detectable transcription factor binding sites (TFBSs), the combination of aligned genomes with the highest base regulatory potential rate (BRPR) value will yield the smaller conserved region (for phylogenetic footprinting algorithm searches). The full list of genome combinations and their BRPR values are given in Additional data file I. The blue line presents the association between percentage of human TFBSs located in conserved regions in a combination of genomes with this BRPR value among all possible genome combinations in this study (see text for detailed description). The grey line plot is similar after the opossum genome is omitted (see text). BRPR, base regulatory potential rate. known human sites in our dataset $(1,162$ sites) in all examined human upstream bases ( 513 genes $\times 5$, ooo bp $=2.565$ megabases), regardless of the species we compare.

Table 3 shows the BRPR values for all comparisons between human and two other species. Interestingly, the highest $B R P R$ value in three species comparisons is achieved when human sequences are compared with both opossum and chicken (BRPR 7.26). However, only 92 of the 1,162 known human TFBSs (7.9\%) may be found via this strategy. Table 3 also shows that requiring a base to be conserved with both mouse and opossum is more effective than using either genome alone, and $31.7 \%$ of known human TFBSs may be detected in this way. The results of all tests (256 combinations) are provided in Additional data file 1. The combination with the overall highest BRPR value was human with chimp, mouse, opossum, and chicken (BRPR 7.628). We note that this maximum BRPR score places a cap on the possible value of $P(R)$. In the unlikely event that all human-chimp-mouseopossum-chicken conserved bases are part of TFBSs (that is, assuming $P(R \mid C)=1$ ), then the maximum value of $P(R)$ from Equation 1 (see materials and methods, below) is (7.628)-1. If we extrapolate, then we find that a maximum of $655 \mathrm{bp}$ may be regulatory in the average human $5 \mathrm{~kb}$ upstream region. Taking the average size of a TFBS in the JASPAR database [35] of high-quality binding sites (10.658 bp) suggests that no more than 61.5 nonoverlapping TFBSs are present in the average $5 \mathrm{~kb}$ upstream region. This maximum value is in agreement with previous reports that estimate this number to be between 10 and 50 sites, depending on the promoter $[36,37]$. The addition of six more (as yet unpublished) vertebrate species in this analysis did not yield a combination of 
Table 3

\begin{tabular}{|c|c|c|c|c|c|c|c|c|}
\hline Human versus & Chimp & Mouse & Rat & Dog & Opossum & Chicken & Fugu & Tetraodon \\
\hline Chimp & & $67.90 \%$ & $62.48 \%$ & $70.65 \%$ & $31.67 \%$ & $8.26 \%$ & $2.75 \%$ & $3.53 \%$ \\
\hline Mouse & 2.896 & & $61.10 \%$ & $59.29 \%$ & $31.67 \%$ & $8.35 \%$ & $2.93 \%$ & $3.79 \%$ \\
\hline Rat & 2.794 & 3.277 & & $54.22 \%$ & $29.43 \%$ & $8.00 \%$ & $2.58 \%$ & $3.44 \%$ \\
\hline Dog & $1.56 \mathrm{I}$ & 3.070 & 2.940 & & $27.54 \%$ & $6.88 \%$ & $2.93 \%$ & $3.79 \%$ \\
\hline Opossum & 5.845 & 6.430 & 6.247 & 5.565 & & $7.92 \%$ & $2.75 \%$ & $3.70 \%$ \\
\hline Chicken & 5.864 & 6.939 & 6.875 & 5.891 & $7.262^{*}$ & & $1.29 \%$ & $1.20 \%$ \\
\hline Fugu & 2.625 & 3.409 & 3.207 & 3.457 & 3.604 & 2.891 & & $2.67 \%$ \\
\hline Tetraodon & 3.195 & 4.103 & 3.951 & 4.165 & 4.620 & 2.775 & 3.468 & \\
\hline
\end{tabular}

Base regulatory potential rate (BRPR) for bases conserved between human and two other species is shown below the diagonal. The rates of transcription factor binding sites detected in blocks conserved between human and two other species are shown above the diagonal. *Highest BRPR value for these 3 -species comparisons.

genomes with a higher BRPR than the human-chimp-mouseopossum-chicken combination (data not shown).

Most phylogenetic footprinting approaches use evolutionary conservation in order to reduce the search space to the parts of the promoters that are more likely to contain functional cis-regulatory elements (for example, see the reports by Sandelin and coworkers [4] and Loots and Ovcharenko [5]). As combinations of more than two genomes are considered, the search space (the jointly conserved region) is reduced. At the same time, the number of sites located within these conserved regions is reduced as well, although at a slower rate. One might then ask, for a given percentage of detectable sites (maximum site sensitivity), which is the combination that minimizes the search space (thereby maximizing specificity)? We found that BRPR scores can be used to address this question. BRPR scores are reversely proportional to $P(C)$, which is the a priori conservation probability (Equation 1; see Materials and methods, below). Thus, the lower the BRPR score, the larger the conserved region and the greater the chance that false-positive TFBS predictions will be made. Therefore, for a given percentage of detectable sites, one wishes to choose the combination of genomes with high BRPR values.

We ranked each of the 1,162 tested human TFBSs according to the highest BRPR value from the combinations of genomes that could detect the given site. From this ranking of sites, it may be seen that some subsets of highly conserved TFBSs may be detected at much higher BRPR thresholds than those sites that are conserved only with closely related species. The proportion of TFBSs that may be detected for a given BRPR threshold is plotted in Figure 4 (blue line). This figure shows, for example, that in order to guarantee detection of $75 \%$ or more of the known TFBSs, one should choose a combination of genomes with BRPR value of 1.7 or less. Naturally, these will be closely related species. By contrast, the combination of genomes with the overall maximum BRPR score (humanchimp-mouse-opossum-chicken, BRPR 7.628) includes only about $7.7 \%$ of the known TFBSs in its conserved regions, whereas the lowest possible BRPR score (human-chimp, BRPR 1.009) includes about 98\%. BRPR values may be more appropriate than evolutionary distance for the purposes of weighting contributions when aiming to discover constrained regulatory sequences in multispecies alignments. We therefore suggest that when it comes to regulatory regions, the BRPR score may be more useful that the 'conservation scores' currently employed in phastCons [38] or MCS [39] approaches.

Figure 4 also shows the importance of including the opossum genome in the comparisons. The grey line displays the same graph, but excluding the opossum genome from the plotted combinations. Without including the opossum genome, the BRPR threshold must be reduced to 3.5 before $20 \%$ of the known TFBSs may be found in the conserved regions. However, with the opossum included, the BRPR threshold for the same search may be increased to 6.5, indicating analogous reduction in the search space. Figure 4 shows that opossum's greatest contribution in terms of phylogenetic footprinting efficiency is for the sensitivity values in the range of $10 \%$ to $33 \%$, although smaller improvements are observed in the $55 \%$ to $65 \%$ range. The 'blocky' nature of the plot is attributable to the subsets of known TFBSs that are detectable in each of the eight species. As more distant mammalian genomes are sequenced, this plot may smooth out to give higher $P(R \mid C)$ scores to more of the known TFBSs.

Our preliminary results including unpublished genomes show that more sites may be predicted with increased BRPR thresholds. Only 20 human sites (1.72\% of known TFBSs) are not detected by any combinatorial approach, suggesting that only a small minority of human TFBSs may not be conserved in any other species. It should also be noted that without the chimp genome, a maximum of $86.5 \%$ of the sites can be identified as conserved, suggesting that only $13.5 \%$ of known human TFBSs may be conserved only among primates. This is an interesting finding, because it establishes $86.5 \%$ as an upper limit to the proportion of TFBSs that may be found 
using traditional phylogenetic footprinting techniques with mouse or more distantly related species. If complete detection of all functional human TFBSs is required, then the phylogenetic shadowing technique for comparing closely related species, proposed by Boffelli and colleagues [40,41], may be more effective than traditional phylogenetic footprinting for primate-specific TFBSs. However, as suggested by those authors, at least six primate genome sequences other than human will be required before phylogenetic shadowing will become effective [40]. Another interesting approach is presented in the recent report by Donaldson and Göttgens [42], which used the mouse genome as an outgroup compared with human and chimpanzee promoters in order to discover regulatory motifs that are conserved in one but not the other [42].

\section{Exploring dependencies between transcription factor binding site nucleotide conservation and the associated transcription factors}

As noted above, the nucleotide conservation within the human TFBSs (as compared with other vertebrates) is higher than the percentage identity in the conserved blocks where they reside (Table 2). This is expected because the regulatory nucleotides may be under stronger evolutionary pressure. Similarly, one would expect that high information content positions (the most conserved positions of the motif) are critical for the binding and thus would also be most conserved across species. This assumption does not take into consideration possible differences in the binding protein residues between species, but it has been shown to be correct for individual yeast and fruit fly transcription factors [43,44]. However, this dependence appears to become weaker when average conservation data are calculated over positions from different vertebrate transcription factors.

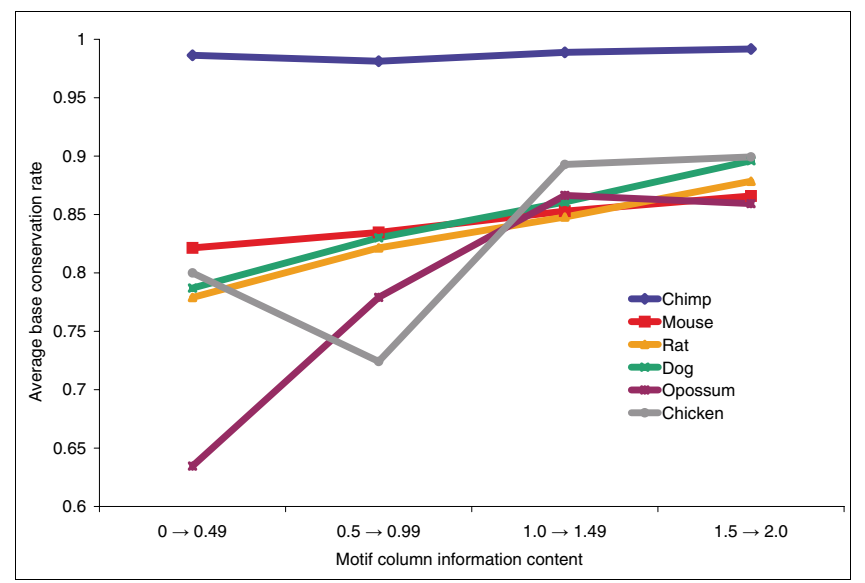

Figure 5

Cross-species conservation of individual TFBS positions versus their information content. Conservation is measured between the human and each of the other species. Information content is measured according to the human position-specific score matrix (PSSM) model.
From the transcription factors included in our dataset, 80 have a position-specific scoring matrix (PSSM) binding model in JASPAR [45] or our manually curated set of mammalian motifs $[6,46]$. These transcription factors are associated with 544 sites in our dataset. The PSSM model of the corresponding transcription factor was used to scan each of its sites from our dataset (see Materials and methods, below). Sometimes the recorded sites extend beyond the length of the PSSM model, reflecting the biochemical method used to discover these sites (for example, DNA footprinting). The highest scoring (sub)sequence was considered to be the correct target site (TFBS), and conservation of each of its nucleotides was calculated for the species in which the site was conserved. The results are plotted in Figure 5, sorted by information content of the corresponding PSSM columns. A weak but definite trend is present in the nonprimate genomes, although even transcription factor motif positions with zero information content (typically assumed to be under no selective pressure) are conserved at a higher rate than the wider conserved blocks. This finding suggests that natural selection operates almost equally strongly across the TFBS positions, regardless of the perceived role of the nucleotide in protein-DNA interactions. One possible explanation for the observed trends is that some motif positions with lower information content may play an indirect role in DNA binding, perhaps by facilitating DNA conformation or by some other mechanism (for instance, Burden and Weng [47] demonstrated conserved DNA structural features at degenerate TFBS locations).

As noted by Sauer and coworkers [11], for human-rodent comparisons certain transcription factors are more likely to have their TFBSs conserved across species than others. We test this finding outside eutherians by examining conservation rates of TFBSs for those factors for which at least seven instances are detectable in the corresponding comparisons. The findings for human-mouse and human-opossum comparisons are presented in Tables 4 and 5, and similar comparisons between human and other species are available in Additional data file 1.

Although some factors' TFBSs are conserved at higher than expected (for example, CREB) or lower than expected (for example, Gfi1, AR and Sp1) rates in human-mouse comparisons, only the sites of Gfi1 are (under)conserved after the Bonferroni correction (see Materials and methods, below). Similarly, the sites of various factors are over-conserved (for example, HMG and CREB, among others) and under-conserved (for example, Gfi1 and Sp1, and so on) in human-opossum comparisons, but only the HMG sites remain (over)conserved after the correction (Table 5). We found that all detectable HMG sites are conserved in both mouse and opossum, but their small number (seven) made them appear significant only in the human-opossum comparisons. Interestingly, human Sp1 TFBSs are under-conserved in all genomes except rodents (Additional data file 1). This may be explained by the fact that the $\mathrm{Sp} 1$ target site (consensus: 
Table 4

Human-mouse TFBS conservation dependency on transcription factor identity

\begin{tabular}{|c|c|c|c|c|c|c|}
\hline \multirow[t]{2}{*}{ Factor } & \multicolumn{2}{|c|}{ Motif } & \multicolumn{4}{|c|}{ Human versus mouse } \\
\hline & IC & Length & Detectable & $\%$ conserved & $p$ value & Over/under \\
\hline HMG & 8.43 & 9 & 7 & $100.00 \%$ & 0.1029 & \\
\hline CREB & 11.52 & 8 & 17 & $94.12 \%$ & 0.0257 & Over \\
\hline c-Myb & 14.15 & 11 & II & $90.91 \%$ & 0.1186 & \\
\hline NF-ATI & N/A & $N / A$ & 10 & $90.00 \%$ & 0.1494 & \\
\hline IPFI & $N / A$ & $\mathrm{~N} / \mathrm{A}$ & 9 & $88.89 \%$ & 0.1862 & \\
\hline $\mathrm{p} 50$ & 15.63 & 11 & 8 & $87.50 \%$ & 0.2292 & \\
\hline NF- $\kappa B$ & 13.34 & 10 & 14 & $85.71 \%$ & 0.1425 & \\
\hline AhR & 8.62 & 6 & 7 & $85.71 \%$ & 0.2775 & \\
\hline GR & 7.06 & 6 & 7 & $85.71 \%$ & 0.2775 & \\
\hline E2F-I & 10.17 & 8 & 12 & $83.33 \%$ & 0.1982 & \\
\hline AP-I & 9.44 & 7 & 34 & $82.35 \%$ & 0.0686 & \\
\hline HIF-I & 11.00 & 11 & 11 & $81.82 \%$ & 0.2286 & \\
\hline MITF & $N / A$ & $N / A$ & II & $81.82 \%$ & 0.2286 & \\
\hline ATF-2 & $N / A$ & $N / A$ & 9 & $77.78 \%$ & 0.2864 & \\
\hline USFI & 10.37 & 6 & 9 & $77.78 \%$ & 0.2864 & \\
\hline $\mathrm{C} / \mathrm{EBP} \alpha$ & 11.12 & 9 & 22 & $77.27 \%$ & 0.1745 & \\
\hline p53 & 25.74 & 18 & 22 & $72.73 \%$ & 0.1897 & \\
\hline $\mathrm{E} 2 \mathrm{~F}$ & 13.84 & 8 & 11 & $72.73 \%$ & 0.2631 & \\
\hline c-Ets-I & $N / A$ & $\mathrm{~N} / \mathrm{A}$ & 7 & $71.43 \%$ & 0.3193 & \\
\hline HNF-I $\alpha$ & N/A & $\mathrm{N} / \mathrm{A}$ & 7 & $71.43 \%$ & 0.3193 & \\
\hline Egr-I & 13.12 & 9 & 12 & $66.67 \%$ & 0.2184 & \\
\hline POUIFIa & 7.57 & 5 & 12 & $66.67 \%$ & 0.2184 & \\
\hline SpI & 9.22 & 8 & 115 & $66.09 \%$ & 0.0250 & Under \\
\hline$H N F-I \alpha-A$ & 13.66 & 10 & 11 & $63.64 \%$ & 0.2010 & \\
\hline GATA-I & 5.57 & 4 & 14 & $57.14 \%$ & 0.1007 & \\
\hline TCF-4 & 12.54 & 7 & 7 & $57.14 \%$ & 0.2032 & \\
\hline EBF & 21.10 & 15 & 8 & $50.00 \%$ & 0.1120 & \\
\hline AP- $-2 \alpha A$ & $N / A$ & $N / A$ & 23 & $47.83 \%$ & 0.0073 & Under \\
\hline ER- $\alpha$ & $N / A$ & $\mathrm{~N} / \mathrm{A}$ & II & $45.45 \%$ & 0.0405 & Under \\
\hline Crx & 11.60 & 10 & 7 & $42.86 \%$ & 0.0772 & \\
\hline Gfil & 7.60 & 4 & 17 & $35.29 \%$ & 0.0012 & Under* \\
\hline$A R$ & $N / A$ & $\mathrm{~N} / \mathrm{A}$ & 7 & $14.29 \%$ & 0.0022 & Under \\
\hline
\end{tabular}

Factors with more than seven sites detectable between the two species are shown. The $p$ values given pertain to the observed percentage of conserved sites, and were determined using the Fisher's exact test. Over/under, specifies over-conservation or under-conservation of the sites of the corresponding transcription factor (by Fisher's exact test) at the $5 \%$ significance level; *Significant under-representation after $p$ value correction (using Bonferroni). Detectable, total number of human transcription factor binding sites located in promoters of mouse orthologous genes; \% conserved, percentage of detectable sites that are in conserved regions; IC, information content (total); Length, length of the motif; N/A, there is no available position-specific score matrix model for this transcription factor; TFBS, transcription factor binding site.

'GGcGGG') and related patterns are expected to occur frequently in GC-rich mammalian promoters. As such, random mutations in mammalian promoters have a high probability of producing additional copies of functional sites. With such a potential proliferation of 'backup' Sp1 target sites, an increased Sp1 TFBS turnover rate should not be surprising. Therefore, evolutionary conservation of TFBSs has some dependency on the identity of the bound transcription factor, but no strong conclusions can be drawn at this point because of the limited amount of available data. AP-2 $\alpha$ is represented by 23 human sites in our dataset. All genes regulated by these sites have orthologs in both mouse and opossum, and yet its TFBSs are under-conserved in mouse. This is an example in which TFBS conservation does not coincide with the conservation of the downstream genes, which has been observed for developmental genes as well [1].

We found no association between the information content (IC) of the transcription factor motif and the percentage conservation. For example, TCF-4 motif has a relatively high IC 
Table 5

\begin{tabular}{|c|c|c|c|c|c|c|}
\hline \multirow[t]{2}{*}{ Factor } & \multicolumn{2}{|c|}{ Motif } & \multicolumn{4}{|c|}{ Human versus opossum } \\
\hline & IC & Length & Detectable & $\%$ conserved & $p$ value & Over/under \\
\hline HMG & 8.43 & 9 & 7 & $100.00 \%$ & 0.0020 & Over* \\
\hline p50 & 15.63 & 11 & 8 & $75.00 \%$ & 0.0470 & Over \\
\hline MITF & $N / A$ & $\mathrm{~N} / \mathrm{A}$ & 10 & $70.00 \%$ & 0.0487 & Over \\
\hline CREB & 11.52 & 8 & 13 & $69.23 \%$ & 0.0287 & Over \\
\hline E2F-I & 10.17 & 8 & 10 & $60.00 \%$ & 0.1228 & \\
\hline GR & 7.06 & 6 & 7 & $57.14 \%$ & 0.2056 & \\
\hline HNF-I $\alpha$ & $\mathrm{N} / \mathrm{A}$ & $\mathrm{N} / \mathrm{A}$ & 7 & $57.14 \%$ & 0.2056 & \\
\hline POUIFIa & 7.57 & 5 & 9 & $55.56 \%$ & 0.1794 & \\
\hline $\mathrm{E} 2 \mathrm{~F}$ & 13.84 & 8 & II & $54.55 \%$ & 0.1594 & \\
\hline AP-I & 9.44 & 7 & 24 & $50.00 \%$ & 0.1112 & \\
\hline ATF-2 & $\mathrm{N} / \mathrm{A}$ & $\mathrm{N} / \mathrm{A}$ & 8 & $50.00 \%$ & 0.2422 & \\
\hline USFI & 10.37 & 6 & 8 & $50.00 \%$ & 0.2422 & \\
\hline IPFI & $N / A$ & N/A & 9 & $44.44 \%$ & 0.2565 & \\
\hline HIF-I & 11.00 & II & 7 & $42.86 \%$ & 0.2938 & \\
\hline p53 & 25.74 & 18 & 16 & $37.50 \%$ & 0.1949 & \\
\hline HNF-I $\alpha-A$ & 13.66 & 10 & 8 & $37.50 \%$ & 0.2763 & \\
\hline$N F-\kappa B$ & 13.34 & 10 & 11 & $36.36 \%$ & 0.2321 & \\
\hline Spl & 9.22 & 8 & 86 & $29.07 \%$ & 0.0049 & Under \\
\hline AP- $2 \alpha A$ & $N / A$ & $N / A$ & 23 & $26.09 \%$ & 0.0581 & \\
\hline $\mathrm{C} / \mathrm{EBP} \alpha$ & 11.12 & 9 & 16 & $25.00 \%$ & 0.0886 & \\
\hline Egr-I & 13.12 & 9 & 8 & $25.00 \%$ & 0.1961 & \\
\hline c-Myb & 14.15 & II & 11 & $18.18 \%$ & 0.0775 & \\
\hline ER- $\alpha$ & $N / A$ & $N / A$ & 9 & $11.11 \%$ & 0.0521 & \\
\hline GATA-I & 5.57 & 4 & 9 & $11.11 \%$ & 0.0521 & \\
\hline Gfil & 7.60 & 4 & II & $0.00 \%$ & 0.0028 & Under \\
\hline AhR & 8.62 & 6 & 7 & $0.00 \%$ & 0.0238 & Under \\
\hline TCF-4 & 12.54 & 7 & 7 & $0.00 \%$ & 0.0238 & Under \\
\hline
\end{tabular}

See Table 5 footnote for details.

value (12.5) and its sites are generally under-conserved in both mouse and opossum, but they are significantly underconserved only in opossum (Tables 4 and 5). In contrast, the sites of HMG are all in conserved regions in human-mouse and human-opossum comparisons, yet the HMG motif has an IC value of 8.4 .

\section{Exploring transcription factor binding site conservation dependencies on Gene Ontology categories between human and opossum}

We also test the possible association between TFBS turnover rates and the functional category of the corresponding regulated genes. Previous studies suggest that the genes with the highest upstream sequence conservation coverage are those involved in transcription and development [48-51]. Table 6 presents the top 30 most populated GO-slim categories [28] in terms of human-mouse orthologous genes from our 513 protein coding gene dataset. Significance was assessed using the Fisher's exact test, as described in the Materials and methods (below). We found that GO categories 'physiologic process' and 'transporter activity' to be over-represented and under-represented, respectively, in both mouse and opossum, even after the Bonferroni correction. Many other GO categories have over-conserved TFBSs in the promoters of their member genes between human and mouse. Examples include 'transcription', 'development', 'cell-cell signaling', response to various stimuli, among others (Table 6). Sauer and coworkers [11] also showed that TFBS conservation in human-rodent comparisons is correlated with the functional category of the downstream regulated gene. Their findings agree with ours in many categories. In particular, there are 34 categories in common for which one (or both) of the studies has found them to be statistically over-represented or underrepresented. In 29 of them (85\%) the two studies agree with respect to the 'sign' of conservation. The differences observed between the two studies can be attributed to the different set 
Table 6

\begin{tabular}{|c|c|c|c|c|c|c|}
\hline GO category & $\begin{array}{l}\text { Number of } \\
\text { genes }\end{array}$ & $\begin{array}{l}\text { Upstream } \\
\text { coverage }\end{array}$ & $\begin{array}{l}\text { Detectable } \\
\text { TFBSs }\end{array}$ & $\begin{array}{l}\% \text { TFBS } \\
\text { detected }\end{array}$ & $p$ value & Over/under \\
\hline Transcription regulator activity & 34 & $37.65 \%$ & 128 & $83.59 \%$ & $6.63 \times 10^{-4}$ & Over* \\
\hline Cell-cell signaling & 44 & $26.00 \%$ & $|4|$ & $82.27 \%$ & $1.27 \times 10^{-3}$ & Over* \\
\hline Development & 55 & $35.19 \%$ & 157 & $81.53 \%$ & $1.41 \times 10^{-3}$ & Over* \\
\hline Nucleotide binding & 42 & $23.31 \%$ & 137 & $79.56 \%$ & $1.04 \times 10^{-2}$ & Over \\
\hline Response to biotic stimulus & 81 & $22.67 \%$ & 273 & $79.49 \%$ & $5.62 \times 10^{-4}$ & Over* \\
\hline Response to external stimulus & 65 & $23.49 \%$ & 209 & $79.43 \%$ & $2.56 \times 10^{-3}$ & Over \\
\hline Response to stress & 91 & $23.78 \%$ & 316 & $79.11 \%$ & $3.50 \times 10^{-4}$ & Over* \\
\hline Physiologic process & 154 & $23.59 \%$ & 526 & $78.90 \%$ & $1.37 \times 10^{-6}$ & Over* \\
\hline Cell proliferation & 53 & $29.13 \%$ & 209 & $78.47 \%$ & $6.00 \times 10^{-3}$ & Over \\
\hline Receptor binding & 65 & $24.36 \%$ & 246 & $77.24 \%$ & $9.74 \times 10^{-3}$ & Over \\
\hline Receptor activity & 42 & $24.55 \%$ & 114 & $77.19 \%$ & $4.29 \times 10^{-2}$ & Over \\
\hline Mitochondrion organization and biogenesis & 100 & $25.26 \%$ & 266 & $77.07 \%$ & $8.93 \times 10^{-3}$ & Over \\
\hline Transcription & 67 & $35.72 \%$ & 223 & $76.68 \%$ & $1.82 \times 10^{-2}$ & Over \\
\hline Extracellular region & 56 & $21.66 \%$ & 217 & $76.04 \%$ & $2.73 \times 10^{-2}$ & Over \\
\hline Protein binding & 142 & $26.43 \%$ & 464 & $75.86 \%$ & $4.75 \times 10^{-3}$ & Over \\
\hline Extracellular space & 54 & $23.08 \%$ & 232 & $75.86 \%$ & $2.70 \times 10^{-2}$ & Over \\
\hline Regulation of biologic process & 155 & $29.96 \%$ & 562 & $75.27 \%$ & $4.97 \times 10^{-3}$ & Over \\
\hline Cytoplasm & 45 & $22.87 \%$ & 136 & $74.26 \%$ & $7.17 \times 10^{-2}$ & \\
\hline Plasma membrane & 57 & $20.12 \%$ & 143 & $74.13 \%$ & $7.10 \times 10^{-2}$ & \\
\hline Transcription factor activity & 42 & $36.92 \%$ & 137 & $73.72 \%$ & $7.62 \times 10^{-2}$ & \\
\hline Nucleus & 92 & $31.28 \%$ & 332 & $73.49 \%$ & $5.00 \times 10^{-2}$ & \\
\hline Cell death & 48 & $21.97 \%$ & 189 & $73.02 \%$ & $6.95 \times 10^{-2}$ & \\
\hline Protein metabolism & 49 & $19.65 \%$ & 147 & $72.79 \%$ & $7.83 \times 10^{-2}$ & \\
\hline Biologic process & 35 & $21.69 \%$ & 100 & $72.00 \%$ & $9.24 \times 10^{-2}$ & \\
\hline Signal transduction & 116 & $23.96 \%$ & 398 & $71.86 \%$ & $5.33 \times 10^{-2}$ & \\
\hline Cell cycle & 41 & $28.45 \%$ & 182 & $70.88 \%$ & $6.34 \times 10^{-2}$ & \\
\hline Cell & 118 & $21.23 \%$ & 351 & $69.23 \%$ & $1.68 \times 10^{-2}$ & Under \\
\hline Binding & 90 & $24.17 \%$ & 297 & $68.69 \%$ & $1.58 \times 10^{-2}$ & Under \\
\hline Transport & 39 & $24.11 \%$ & 146 & $67.81 \%$ & $3.30 \times 10^{-2}$ & Under \\
\hline Catalytic activity & 40 & $19.68 \%$ & 99 & $61.62 \%$ & $4.63 \times 10^{-3}$ & Under \\
\hline Transporter activity & 35 & $25.00 \%$ & 123 & $60.98 \%$ & $1.20 \times 10^{-3}$ & Under* \\
\hline
\end{tabular}

The top 31 Gene Ontology (GO) categories in terms of gene numbers in the dataset are shown. The $p$ values given represent the significance (uncorrected) of the observed percentage of conserved (detected) sites, as determined using the Fisher's exact test. Over/under, specifies overconservation or under-conservation of the sites of the corresponding GO category (by Fisher's exact test) at the $5 \%$ significance level. *Statistical over-representation or under-representation after $p$ value correction (using Bonferroni). TFBS, transcription factor binding site.

of TFBSs upon which their measurements are based (Sauer and coworkers used sites from mouse and rat in addition to human) and the methods used to assign significance.

We extend this study in opossum (Table 7) and the other vertebrate genomes (Additional data file 1). Most of the overconserved categories between human and mouse are also over-conserved in human-opossum comparisons (Fisher's exact test; see Tables 6 and 7). These include 'cell-cell signaling' and response to stress and biotic stimuli. On the other hand, the TFBS conservation rate for the 'protein binding' went from being over-conserved in human-mouse comparisons ( $76 \%$ TFBS conservation) to under-conserved in humanopossum comparisons ( $36 \%$ TFBS conservation). This is one of the highly populated categories, and its members are involved in almost every cellular process, for instance signal transduction, chromatin structure, transcription, translation, cell cytoskeleton, and so on. It is therefore difficult to assess the significance of this change in TFBS conservation related to this category. One thing is for sure; the observed differences are not an artifact caused by the low number of TFBSs. This category is represented by 142 genes associated with 464 TFBSs in mouse and 122 genes associated with 419 TFBSs in opossum, making it one of the best represented categories in our dataset.

'Development' is another category in which TFBSs are significantly over-conserved in human-mouse comparisons. In 
Table 7

\begin{tabular}{|c|c|c|c|c|c|c|}
\hline GO category & $\begin{array}{l}\text { Number of } \\
\text { genes }\end{array}$ & $\begin{array}{l}\text { Upstream } \\
\text { Coverage }\end{array}$ & $\begin{array}{l}\text { Detectable } \\
\text { TFBSs }\end{array}$ & $\begin{array}{l}\text { \% TFBS } \\
\text { Detected }\end{array}$ & $p$ value & Over/under \\
\hline Receptor binding & 51 & $6.49 \%$ & 180 & $55.56 \%$ & $5.80 \times 10^{-6}$ & Over* \\
\hline Cell-cell signaling & 35 & $6.37 \%$ & 120 & $51.67 \%$ & $3.67 \times 10^{-3}$ & Over \\
\hline Physiologic process & 122 & $5.63 \%$ & 415 & $49.40 \%$ & $1.51 \times 10^{-6}$ & Over* \\
\hline Response to external stimulus & 54 & $5.60 \%$ & 168 & $48.81 \%$ & $6.12 \times 10^{-3}$ & Over \\
\hline Transcription regulator activity & 32 & $10.15 \%$ & 122 & $47.54 \%$ & $2.47 \times 10^{-2}$ & Over \\
\hline Extracellular space & 43 & $4.08 \%$ & 175 & $47.43 \%$ & $1.23 \times 10^{-2}$ & Over \\
\hline Response to biotic stimulus & 60 & $5.29 \%$ & 209 & $47.37 \%$ & $7.82 \times 10^{-3}$ & Over \\
\hline Transcription & 61 & $10.52 \%$ & 208 & $45.67 \%$ & $2.13 \times 10^{-2}$ & Over \\
\hline Transcription factor activity & 40 & $9.80 \%$ & 133 & $45.11 \%$ & $4.65 \times 10^{-2}$ & Over \\
\hline Development & 47 & $9.48 \%$ & 120 & $45.00 \%$ & $5.25 \times 10^{-2}$ & \\
\hline Signal transduction & 86 & $5.72 \%$ & 293 & $44.71 \%$ & $1.95 \times 10^{-2}$ & Over \\
\hline Response to stress & 74 & $6.23 \%$ & 268 & $44.03 \%$ & $3.18 \times 10^{-2}$ & Over \\
\hline Regulation of biologic process & 134 & $8.49 \%$ & 490 & $43.06 \%$ & $2.59 \times 10^{-2}$ & Over \\
\hline Cell & 82 & $6.11 \%$ & 241 & $40.66 \%$ & $5.96 \times 10^{-2}$ & \\
\hline Nucleus & 81 & $10.05 \%$ & 305 & $40.66 \%$ & $5.52 \times 10^{-2}$ & \\
\hline Extracellular region & 44 & $6.17 \%$ & 160 & $40.63 \%$ & $6.95 \times 10^{-2}$ & \\
\hline Cell proliferation & 49 & $7.63 \%$ & 196 & $40.31 \%$ & $6.26 \times 10^{-2}$ & \\
\hline Mitochondrion organization and biogenesis & 77 & $6.90 \%$ & 213 & $39.44 \%$ & $5.29 \times 10^{-2}$ & \\
\hline Cytoplasm & 34 & $6.07 \%$ & 97 & $39.18 \%$ & $7.95 \times 10^{-2}$ & \\
\hline Cell death & 41 & $6.77 \%$ & 164 & $37.80 \%$ & $4.34 \times 10^{-2}$ & Under \\
\hline Protein binding & 122 & $7.01 \%$ & 419 & $35.80 \%$ & $4.81 \times 10^{-4}$ & Under* \\
\hline Cell cycle & 39 & $7.67 \%$ & 176 & $35.23 \%$ & $1.35 \times 10^{-2}$ & Under \\
\hline Nucleotide binding & 32 & $5.82 \%$ & 112 & $31.25 \%$ & $5.81 \times 10^{-3}$ & Under \\
\hline Protein complex & 28 & $5.90 \%$ & 84 & $29.76 \%$ & $7.37 \times 10^{-3}$ & Under \\
\hline DNA binding & 27 & $10.05 \%$ & 74 & $29.73 \%$ & $1.08 \times 10^{-2}$ & Under \\
\hline Binding & 7I & $6.77 \%$ & 240 & $29.58 \%$ & $5.58 \times 10^{-6}$ & Under* \\
\hline Receptor activity & 31 & $6.53 \%$ & 88 & $29.55 \%$ & $5.67 \times 10^{-3}$ & Under \\
\hline Plasma membrane & 37 & $4.78 \%$ & 91 & $28.57 \%$ & $3.00 \times 10^{-3}$ & Under \\
\hline Protein metabolism & 41 & $6.27 \%$ & 131 & $25.95 \%$ & $3.75 \times 10^{-5}$ & Under* \\
\hline Transporter activity & 31 & $6.28 \%$ & 91 & $23.08 \%$ & $6.74 \times 10^{-5}$ & Under* \\
\hline Transport & 32 & $5.53 \%$ & 102 & $20.59 \%$ & $1.85 \times 10^{-6}$ & Under* \\
\hline
\end{tabular}

See Table 6 footnote for details.

human-opossum comparisons TFBSs are still over-conserved, but not at a significant level. This can also be attributed to the sharp decrease in the percentage of detected TFBSs (from $81.5 \%$ in mouse to $45 \%$ in opossum) in relation to the high number of potentially detectable TFBSs (157 versus 120 in mouse and opossum, respectively). The developmental genes themselves are ultra-conserved in opossum [1], resulting in the detection of many orthologs and hence many potentially detectable TFBSs associated with them. Conservation tables, similar to Tables 6 and 7, for comparisons between human and other species are available in Additional data file 1.

\section{Comparison with other studies}

A number of existing studies have attempted to quantify regulatory conservation in mammals, albeit using different approaches and typically restricting their interest to humanrodent comparisons. Our results on human-rodent comparisons generally agree with these studies. For example, we find approximately $72 \%$ of detectable human TFBSs conserved in mouse $5 \mathrm{~kb}$ upstream regions. Similarly, Sauer and coworkers [11] reported detection of TRANSFAC [29] TFBSs in humanrodent conserved sequences at a rate of $71.7 \%$ when using the same conservation threshold (65\% identity).

For conservation cutoffs of 70\% identity, Liu and coworkers [9], Levy and Hannenhalli [12], and Lenhard and colleagues [13] independently found human-mouse conservation rates for known TFBSs of about $60 \%, 65 \%$, and $68 \%$, respectively. The latter three studies were also based on finding conserved blocks via sliding windows on aligned sequences. Dermitzakis and Clark [10] also reported detection of TRANSFAC TFBSs 
in human-rodent conserved sequences at rates of $60 \%$ to $68 \%$. All of the aforementioned human-rodent TFBS turnover rates are consistent with our findings, given the slightly higher conservation cut-offs and the lower number of known TFBSs tested (40 sites by Lenhard and colleagues [13], 64 sites by Dermitzakis and Clark [10], 467 sites by Liu and coworkers [9], and 481 sites by Levy and Hannenhalli [12]).

In relation to our human-mouse $5 \mathrm{~kb}$ upstream conservation coverage figure (24\%), a number of other studies have found human-rodent upstream conservation rates in the range $17 \%$ to $25 \%[9,52,53]$. In a comparison of 77 well defined humanmouse gene pairs, Jareborg and coworkers [54] found $36 \%$ conservation coverage of upstream sequence using the software program DBA and a $60 \%$ cutoff. However, their upstream sequences ranged from $500 \mathrm{bp}$ to $1,000 \mathrm{bp}$ upstream of the TSS. Our conservation coverage in the same range of distance is $38.7 \%$ to $49.2 \%$. Sauer and coworkers [11] found a background conservation rate of $35 \%$ in humanrodent comparisons, although their study was based on 800 bp windows of sequence centered on a known TFBSs, and was therefore also biased toward including sequence from the proximal 500 bp region.

A recent study of the mouse transcriptome showed that a large part of this mammalian genome may be transcribed [55]. The authors found many more transcripts than the number of genes currently estimated for the mammalian genomes. For about one-third of these transcripts no association with protein coding genes was found, and therefore they were considered to be noncoding RNAs (ncRNAs). Similar to our study, the authors analyzed the upstream sequences of these potential ncRNAs, which they found to be more conserved than the promoters of the protein coding genes. However, their study has some differences compared with ours. First, it does not focus specifically on the intergenic miRNA genes, but analyzes all transcripts for which no protein coding gene association was found. Also, their study does not depict the similarity we found in the conservation rates of coding and noncoding upstream regions in the first $500 \mathrm{bp}$, which is an important finding of our study, especially when compared with the conservation of the upstream sequences of the tRNA genes (Figure 2). Cooper and coworkers [56] recently analyzed the conservation rates of core promoter sequences of protein coding genes. Their findings agree with ours in that they find that the first $300 \mathrm{bp}$ upstream of the TSS are important for the core promoter activity. This is the region where we find the highest conservation (Figure 2). In another study, Taylor and coworkers [57] reported that the nucleotide substitution rate increases with the distance from TSS in various types of protein coding genes in a way similar to our observations.

\section{Conclusion}

This study is the first to analyze conservation of the upstream regions of protein coding genes in relation to the upstream regions of intergenic miRNA genes. We found the latter to be about twice as conserved as the former beyond the first 500 bp. The reason for this conservation is currently unknown. The first $500 \mathrm{bp}$ appear to be equally conserved in both types of genes, a feature that is missing from the upstream sequences of the tRNA genes. This indicates that similar mechanisms of gene regulation may be in place, which is in agreement with other studies [27,32]. The difference in conservation rates is more apparent in the mammalian lineages, including opossum, and may reflect similarities in mammalian gene regulation.

Another important finding is that the opossum genome offers great potential in terms of improving the performance of the phylogenetic footprinting algorithms. We found that $41 \%$ of the known human TFBSs are located in the $6.7 \%$ of promoter regions that are conserved between human and opossum, illustrating that the opossum genome sequence can be used to reduce the search space for a large proportion of human TFBSs. A new statistical measure, BRPR, is introduced that quantifies the trade-off between sequence conservation (or reduction of the search space for comparative genomics strategies) and regulatory site conservation. We show that for a given site sensitivity threshold, an appropriate combination of genomes can be selected to minimize the search space. Finally, we find that basic cellular functions, such as cell-cell signaling and receptor binding, have significantly over-conserved sites between human and opossum (the corresponding genes have more TFBSs located in the conserved parts of their promoter regions). By contrast, TFBSs related to functions such as transporter activity and protein metabolism are significantly under-conserved.

\section{Materials and methods MicroRNA gene dataset}

Human miRNA genes were retrieved from the miRBase [58] and the UCSC Genome Browser (version hg18, March 2006) [59]. Cross-referencing them with the miRNAMap dataset [6o] identified 169 putatively intergenic miRNA genes. The sequences of these miRNAs were used in BLAST-like Alignment Tool (BLAT) [61] alignments against the latest UCSC human genome and their exact genomic locations were identified. Following observations in previous studies [27,62], we consider two miRNA genes to be co-transcribed if their starting points are less than $250 \mathrm{bp}$ apart. In this way, we identified 12 clusters containing 31 genes. Only the 5 '-most gene in a cluster was considered in our analysis. Five miRNA genes were found to reside within large introns of protein coding genes, and although they may have their own regulatory regions, we excluded them from further analysis. This resulted in a dataset of 145 human intergenic miRNA genes (Additional data file 1). The coordinates of the BLAT outputs 
were used to retrieve up to $5 \mathrm{~kb}$ regions upstream of the gene start site as described below.

We note that in a recent study, Devor and Samollow (personal communication) tested 71 predicted miRNA genes using quantitative polymerase chain reaction on pooled RNA from brain, heart, lung, liver, tongue, and esophagus from an adult opossum. They found evidence of expression in $80 \%$ of the cases they tested, including 36 genes in our opossum dataset.

\section{Pair-wise and multiple species comparisons}

Pair-wise and multiple species alignments for both protein coding and miRNA genes were retrieved from the 17-species MULTIZ multiple alignments [38], which are available from the UCSC web server (version hg18, March 2006) [63]. The MULTIZ algorithm builds a multiple alignment from local pair-wise BLASTZ alignments of the reference genome with each other genome of interest [38,64]. Each base in the reference genome is aligned to at most one base in the other genomes, and the alignment is guided by synteny. In this study, we present the results from pair-wise and multiple species comparisons of human [65] with four eutherian mammals (chimpanzee [66], mouse [67], rat [68], and dog [15]), the newly sequenced opossum [1], chicken [69], fugu [70], and tetraodon [71]. A phylogenetic tree for those species and with branch lengths derived from the ENCODE project Multi-Species Sequence Analysis group (September 2005) is shown in Figure 1. This tree was generated using the phyloGif program [72] from Threaded Blockset Aligner (TBA) alignments over 23 vertebrate species and is based on $4 \mathrm{D}$ sites (similar to the tree presented by Margulies and coworkers [73]).

For each pair-wise or multiple species comparisons, the corresponding (aligned) $5 \mathrm{~kb}$ upstream sequences were retrieved directly from the MULTIZ alignments for greater accuracy, using the human genes as reference. If other genes were found within this $5 \mathrm{~kb}$ range, then the upstream sequences were shortened accordingly to exclude the additional genes. We used the $65 \%$ as our conserved block threshold, which is similar to that in previous studies $[9,12,13]$ and similar to the default threshold used by many phylogenetic footprinting algorithms [6,13].

\section{tRNA dataset}

Human tRNA genes and pair-wise alignments were extracted from the UCSC Genome Browser database (version hg18, March 2006) using the genomic MULTIZ alignments as we describe above. Genes that were found to be facing opposite directions in the genome ('head-to-head') and their starts were closer than $2.5 \mathrm{~kb}$ apart were excluded from the analysis. This rule excluded 156 genes. The final human tRNA dataset included 1,795 upstream sequences.

\section{Dataset of known transcription factor binding sites}

TRANSFAC database (release 9.3) [29] contains 1,162 human confirmed TFBSs that satisfy the following criteria: the site is experimentally confirmed and associated with a promoter of a human gene from the database (confirmed sites); the TFBS sequence can be found within $5 \mathrm{~kb}$ upstream of the TSS; if multiple site occurrences are present in the corresponding promoter, then positional information (relative to TSS) is listed in the database; and the regulated human gene corresponds to an entry in the RefSeq gene collection. The above TFBSs are located in the promoters of 513 human genes, which serves as our primary dataset for the transcription factor-TFBS association study. We focus on the sites located in the $5 \mathrm{~kb}$ upstream region, because this includes $83.4 \%$ of all known human TFBSs in TRANSFAC (data not shown). The majority of the sites (a total of 774) have a TRANSFAC assigned quality score of $1,2,3$, or 4 , which shows confirmed binding activity to a known transcription factor. For an additional 325 sites, no TRANSFAC quality score was assigned. The remaining 63 sites (about $5 \%$ ) belong to TRANSFAC category 5 , for which an unknown protein has been shown to bind to a DNA element.

\section{Dataset of position-specific scoring matrix models}

JASPAR database [35] contains 20 PSSM models for transcription factors whose sites are present in our dataset. In addition, we previously generated manually 60 more PSSM models from high-quality human and mouse sites in TRANSFAC [6], which we make publicly available through our web server [74]. These models were used to analyze the position information content with the nucleotide conservation in the subset of 572 corresponding known TFBSs (Figure 5).

\section{Conserved blocks and transcription factor binding site detection: some definitions}

In this study, sequence conservation is expressed as conserved block coverage. A sliding window of width 50 bp and step size 10 bp was used to find conserved regions (or blocks) of at least $65 \%$ identity between human and each other species. Each pair-wise alignment was extracted from the MULTIZ multiple alignments. Sauer and coworkers [11] have shown that the $65 \%$ identity threshold most effectively separates TFBSs from background sequence in human-rodent comparisons. The percentage of human $5 \mathrm{~kb}$ upstream sequence that is located within conserved blocks is denoted the 'conserved block coverage'. The 'average block conservation' is the percentage of identical bases in conserved blocks over all bases in conserved blocks. A 'conserved site' is a known human TFBS that overlaps a conserved block between human and another species. Because we explore the effect of sequence and pattern of conservation in the discovery of cisregulatory elements, this study does not make any assumptions about the biologic functionality of the human-equivalent TFBSs in the other organisms. In other words, we cannot address the issue of actual site turnover, but simply whether a known human TFBS is located in a conserved block between 
human and one or more other species (regardless of whether it is functional in these other species). 'Detectable TFBSs' are those sites that are in the promoters of genes that have orthologs in the other species (in terms of UCSC multispecies alignments). A detectable site is considered to be 'conserved' between two species if it is located in a conserved block in their corresponding pair-wise alignment. When multiple species are considered, a TFBS is considered to be conserved if it is conserved in each of the species. The 'TFBS conservation rate' between human and other species is defined as the percentage of detectable TFBSs found to be conserved. The conservation rate can be thought of as the upper limit of sensitivity (at the site level) of a phylogenetic footprinting algorithm if only the conserved regions are analyzed. Such algorithms include ConSite and rVista [13,75]. In general, the methods and thresholds used to define conserved blocks were chosen to reflect those typically used by phylogenetic footprinting algorithms $[6,13,46,75]$ and by other researchers [9,11-13].

\section{Base regulatory potential rate}

A base position is called 'regulatory' if it is part of a TFBS. For this report, bases in nonhuman species that are aligned to human regulatory bases are also called regulatory. We understand that this definition is only made for the purposes of this analysis and does not imply any functional role. However, it is expected that the majority of known human sites that are conserved in various species would also be functional in these species. Given a promoter alignment between two species, we define the base regulatory potential rate (BRPR) as the conditional probability of a base being regulatory given it is located in a conserved region over the prior probability of being regulatory. Formally, BRPR is defined in the first part of the following equation:

$$
\mathrm{BRPR}=\frac{P(R \mid C)}{P(R)}=\frac{P(C \mid R)}{P(C)}
$$

where $R$ denotes the base as regulatory (part of a known human TFBS) and $C$ indicates that it is located in a conserved region. The last part of the equation derives from the Bayesian rule and is the one we use for the calculation of BRPR because $P(R \mid C)$ cannot be reliably estimated, given our limited knowledge of mammalian TFBSs. In other words, BRPR shows how much we improve our regulatory potential prediction if we restrict our search space to conserved regions only. $P(C)$ and $P(C \mid R)$ are directly estimated from the data. $P(R)$ is the $a$ priori probability of a base being regulatory in a given promoter, and it depends on the size of the promoter as well as the number and size of cis-regulatory elements found within. According to our current knowledge of transcriptional control, $P(R)$ decreases as one examines windows of sequence more distal to the transcription start site. In this way, calculated BRPR values are dependant on the length of upstream sequence examined from the transcription start. BRPR values decrease as the examined regions become smaller ( $5 \mathrm{~kb}$ to 1 $\mathrm{kb}$ or $500 \mathrm{bp}$ from the TSS; Additional data file 1 [Supplementary Figure 1]) because, from Equation 1 above, $P(R)$ increases in these shorter regions while $P(R \mid C)$ remains relatively constant. The important point to note, however, is that the relative BRPR rankings of different genome combinations remain constant (Additional data file 1 [Supplementary Figure 1]).

\section{Assessing significance of over-conservation or under- conservation for sets of transcription factor binding sites}

The Fisher's exact test on $2 \times 2$ contingency tables is used to estimate the significance of under-conservation or over-conservation of sites bound by particular transcription factors or associated with certain GO categories (Tables 4 to 7 ). To account for multiple testing we applied the Bonferroni correction, although the data dependencies among the tests make that correction slightly conservative. Statistically over-represented and under-represented categories are presented in Tables 4 to 7 in the corresponding column, and those values that remain significant after the Bonferroni correction are marked with asterisks.

\section{Additional data files}

The following additional data are available with the online version of this paper. Additional data file 1 provides various descriptions, generalized analyses, and supplementary data that complement and extend those given in the main text.

\section{Acknowledgements}

We would like to thank Paul Samollow for being the driving force behind the idea of sequencing Monodelphis. We also thank the people from the Broad Institute for their efforts in sequencing and annotating the Monodelphis genome. Special thanks go to Kerstin Lindblad-Toh, Michael Zody, Tarjei Mikkelsen, and Candace Kammerer for helpful discussions. We also thank two anonymous reviewers for their comments that helped us to improve the manuscript. This work was supported by $\mathrm{NIH}$ grants RROI42I4 and NOI Al-500I8, NSF grant MCB03I6255 and a grant with the Pennsylvania Department of Health. The PA Department of Health specifically disclaims responsibility for any analyses, interpretations or conclusions. PVB was also supported by $\mathrm{NIH}$ grant IROILM007994-0I and TATRC/DoD USAMRAA Prime Award W8IXWH-05-2-0066.

\section{References}

I. Mikkelsen TS, Wakefield MJ, Aken B, Amemiya CT, Chang JL, Duke S, Garber M, Gentles AJ, Goodstadt L, Heger A, et al.: Genome of the marsupial Monodelphis domestica reveals innovation in noncoding sequences. Nature 2007, 447:167-178.

2. Belov K, Deakin JE, Papenfuss AT, Baker ML, Melman SD, Siddle HV, Gouin N, Goode DL, Sargeant TJ, Robinson MD, et al.: Reconstructing an ancestral mammalian immune supercomplex from a marsupial major histocompatibility complex. PLoS Biol 2006 4:e46.

3. Siddharthan R, Siggia ED, van Nimwegen E: PhyloGibbs: a Gibbs sampling motif finder that incorporates phylogeny. PLoS Comput Biol 2005, I:e67.

4. Sandelin A, Wasserman WW, Lenhard B: ConSite: web-based prediction of regulatory elements using cross-species comparison. Nucleic Acids Res 2004:W249-W252.

5. Loots GG, Ovcharenko I: rVISTA 2.0: evolutionary analysis of transcription factor binding sites. Nucleic Acids Res 
2004:W2|7-22|

6. Corcoran DL, Feingold E, Dominick J, Wright M, Harnaha J, Trucco M, Giannoukakis N, Benos PV: Footer: a quantitative comparative genomics method for efficient recognition of cis-regulatory elements. Genome Res 2005, 15:840-847.

7. Stormo GD: DNA binding sites: representation and discovery. Bioinformatics 2000, 16:16-23.

8. Wasserman WW, Sandelin A: Applied bioinformatics for the identification of regulatory elements. Nat Rev Genet 2004, 5:276-287.

9. Liu Y, Liu XS, Wei L, Altman RB, Batzoglou S: Eukaryotic regulatory element conservation analysis and identification using comparative genomics. Genome Res 2004, 14:45I-458.

10. Dermitzakis ET, Clark AG: Evolution of transcription factor binding sites in Mammalian gene regulatory regions: conservation and turnover. Mol Biol Evol 2002, 19: I I I4-II2I.

II. Sauer T, Shelest E, Wingender E: Evaluating phylogenetic footprinting for human-rodent comparisons. Bioinformatics 2006, 22:430-437.

12. Levy S, Hannenhalli S: Identification of transcription factor binding sites in the human genome sequence. Mamm Genome 2002, 13:5|0-5|4.

13. Lenhard B, Sandelin A, Mendoza L, Engstrom P, Jareborg N, Wasserman WW: Identification of conserved regulatory elements by comparative genome analysis. J Biol 2003, 2: 13.

14. Murphy WJ, Pevzner PA, O'Brien SJ: Mammalian phylogenomics comes of age. Trends Genet 2004, 20:631-639.

15. Lindblad-Toh K, Wade CM, Mikkelsen TS, Karlsson EK, Jaffe DB, Kamal M, Clamp M, Chang JL, Kulbokas EJ III, Zody MC, et al.: Genome sequence, comparative analysis and haplotype structure of the domestic dog. Nature 2005, 438:803-819.

16. Lee RC, Feinbaum RL, Ambros V: The C. elegans heterochronic gene lin-4 encodes small RNAs with antisense complementarity to lin- I4. Cell 1993, 75:843-854.

17. Ambros V: The functions of animal microRNAs. Nature 2004, 431:350-355.

18. John B, Enright AJ, Aravin A, Tuschl T, Sander C, Marks DS: Human MicroRNA targets. PLoS Biol 2004, 2:e363.

19. Lewis BP, Shih IH, Jones-Rhoades MW, Bartel DP, Burge CB: Prediction of mammalian microRNA targets. Cell 2003, I I 5:787-798.

20. Kiriakidou M, Nelson PT, Kouranov A, Fitziev P, Bouyioukos C, Mourelatos Z, Hatzigeorgiou A: A combined computationalexperimental approach predicts human microRNA targets. Genes Dev 2004, 18: I I65-I I78.

21. Bartel DP, Chen CZ: Micromanagers of gene expression: the potentially widespread influence of metazoan microRNAs. Nat Rev Genet 2004, 5:396-400.

22. Chen CZ, Li L, Lodish HF, Bartel DP: MicroRNAs modulate hematopoietic lineage differentiation. Science 2004, 303:83-86.

23. Farh KK, Grimson A, Jan C, Lewis BP, Johnston WK, Lim LP, Burge $\mathrm{CB}$, Bartel DP: The widespread impact of mammalian MicroRNAs on mRNA repression and evolution. Science 2005, 310:1817-182|.

24. Lee CT, Risom T, Strauss WM: MicroRNAs in mammalian development. Birth Defects Res C Embryo Today 2006, 78: I29-139.

25. Krichevsky AM, King KS, Donahue CP, Khrapko K, Kosik KS: A microRNA array reveals extensive regulation of microRNAs during brain development. RNA 2003, 9:1274-I28I.

26. Taganov KD, Boldin MP, Chang KJ, Baltimore D: NF-kappaBdependent induction of microRNA miR-146, an inhibitor targeted to signaling proteins of innate immune responses. Proc Natl Acad Sci USA 2006, 103: | 248I- 12486.

27. Lee Y, Kim M, Han J, Yeom KH, Lee S, Baek SH, Kim VN: MicroRNA genes are transcribed by RNA polymerase II. EMBO J 2004, 23:405I-4060

28. Harris MA, Clark J, Ireland A, Lomax J, Ashburner M, Foulger R, Eilbeck K, Lewis S, Marshall B, Mungall C, et al:: The Gene Ontology (GO) database and informatics resource. Nucleic Acids Res 2004:D258-D26I.

29. Matys V, Fricke E, Geffers R, Gossling E, Haubrock M, Hehl R, Hornischer K, Karas D, Kel AE, Kel-Margoulis OV, et al:: TRANSFAC: transcriptional regulation, from patterns to profiles. Nucleic Acids Res 2003, $31: 374-378$

30. Nichols M, Bell J, Klekamp MS, Weil PA, Soll D: Multiple mutations of the first gene of a dimeric tRNA gene abolish in vitro tRNA gene transcription. J Biol Chem 1989, 264:17084-I7090.

31. Leaman D, Chen PY, Fak J, Yalcin A, Pearce M, Unnerstall U, Marks DS, Sander C, Tuschl T, Gaul U: Antisense-mediated depletion reveals essential and specific functions of microRNAs in Drosophila development. Cell 2005, I 2 I:1097-I I08.

32. Johnston RJ Jr, Hobert O: A novel C. elegans zinc finger transcription factor, Isy-2, required for the cell type-specific expression of the Isy-6 microRNA. Development 2005, 132:545I-5460.

33. Fisher S, Grice EA, Vinton RM, Bessling SL, McCallion AS: Conservation of RET regulatory function from human to zebrafish without sequence similarity. Science 2006, 3 | 2:276-279.

34. Ludwig MZ, Bergman C, Patel NH, Kreitman M: Evidence for stabilizing selection in a eukaryotic enhancer element. Nature 2000, 403:564-567.

35. Vlieghe D, Sandelin A, De Bleser PJ, Vleminckx K, Wasserman WW, van Roy F, Lenhard B: A new generation of JASPAR, the openaccess repository for transcription factor binding site profiles. Nucleic Acids Res 2006:D95-D97.

36. Arnone MI, Davidson $\mathrm{EH}$ : The hardwiring of development: organization and function of genomic regulatory systems. Development 1997, 124:185I-1864.

37. Hanson RW, Reshef L: Regulation of phosphoenolpyruvate carboxykinase (GTP) gene expression. Annu Rev Biochem 1997, 66:58I-6III.

38. Siepel A, Bejerano G, Pedersen JS, Hinrichs AS, Hou M, Rosenbloom K, Clawson H, Spieth J, Hillier LW, Richards S, et al.: Evolutionarily conserved elements in vertebrate, insect, worm, and yeast genomes. Genome Res 2005, I 5:1034-1050.

39. Margulies EH, Blanchette M, Haussler D, Green ED: Identification and characterization of multi-species conserved sequences. Genome Res 2003, 13:2507-25 I8.

40. Boffelli D, McAuliffe J, Ovcharenko D, Lewis KD, Ovcharenko I, Pachter L, Rubin EM: Phylogenetic shadowing of primate sequences to find functional regions of the human genome. Science 2003, 299:।39|-I394.

4I. Ovcharenko I, Boffelli D, Loots GG: eShadow: a tool for comparing closely related sequences. Genome Res 2004, 14:1 I91-I 198.

42. Donaldson IJ, Gottgens B: Evolution of candidate transcriptional regulatory motifs since the human-chimpanzee divergence. Genome Biol 2006, 7:R52.

43. Moses AM, Chiang DY, Kellis M, Lander ES, Eisen MB: Position specific variation in the rate of evolution in transcription factor binding sites. BMC Evol Biol 2003, 3:19.

44. Moses AM, Pollard DA, Nix DA, lyer VN, Li XY, Biggin MD, Eisen MB Large-scale turnover of functional transcription factor binding sites in Drosophila. PLoS Comput Biol 2006, 2:el 30.

45. Sandelin A, Alkema W, Engstrom P, Wasserman WW, Lenhard B. JASPAR: an open-access database for eukaryotic transcription factor binding profiles. Nucleic Acids Res 2004:D9I-D94.

46. Corcoran DL, Feingold E, Benos PV: FOOTER: a web tool for finding mammalian DNA regulatory regions using phylogenetic footprinting. Nucleic Acids Res 2005:W442-W446.

47. Burden HE, Weng Z: Identification of conserved structural features at sequentially degenerate locations in transcription factor binding sites. Genome Inform 2005, 16:49-58.

48. Bejerano G, Pheasant M, Makunin I, Stephen S, Kent WJ, Mattick JS, Haussler D: Ultraconserved elements in the human genome. Science 2004, 304: 1321-1325.

49. Iwama $H$, Gojobori T: Highly conserved upstream sequences for transcription factor genes and implications for the regulatory network. Proc Natl Acad Sci USA 2004, I0 I: 17156-17161.

50. Sandelin A, Bailey P, Bruce S, Engstrom PG, Klos JM, Wasserman WW, Ericson J, Lenhard B: Arrays of ultraconserved non-coding regions span the loci of key developmental genes in vertebrate genomes. BMC Genomics 2004, 5:99.

51. Woolfe A, Goodson M, Goode DK, Snell P, McEwen GK, Vavouri T, Smith SF, North P, Callaway H, Kelly K, et al.: Highly conserved non-coding sequences are associated with vertebrate development. PLoS Biol 2005, 3:e7.

52. Shabalina SA, Ogurtsov AY, Kondrashov VA, Kondrashov AS: Selective constraint in intergenic regions of human and mouse genomes. Trends Genet 200I, 17:373-376.

53. Wasserman WW, Palumbo M, Thompson W, Fickett JW, Lawrence CE: Human-mouse genome comparisons to locate regulatory sites. Nat Genet 2000, 26:225-228.

54. Jareborg N, Birney E, Durbin R: Comparative analysis of noncoding regions of 77 orthologous mouse and human gene pairs. Genome Res 1999, 9:815-824.

55. Carninci P, Kasukawa T, Katayama S, Gough J, Frith MC, Maeda N, Oyama R, Ravasi T, Lenhard B, Wells C, et al.: The transcriptional 
landscape of the mammalian genome. Science 2005, 309:1559-1563.

56. Cooper SJ, Trinklein ND, Anton ED, Nguyen L, Myers RM: Comprehensive analysis of transcriptional promoter structure and function in $1 \%$ of the human genome. Genome Res 2006, 16:1-10.

57. Taylor MS, Kai C, Kawai J, Carninci P, Hayashizaki Y, Semple CA: Heterotachy in mammalian promoter evolution. PLoS Genet 2006, 2:e30.

58. Griffiths-Jones S, Grocock RJ, van Dongen S, Bateman A, Enright AJ: miRBase: microRNA sequences, targets and gene nomenclature. Nucleic Acids Res 2006:D I 40-D I44.

59. UCSC Genome Browser [http://genome.ucsc.edu/]

60. Hsu PW, Huang HD, Hsu SD, Lin LZ, Tsou AP, Tseng CP, Stadler PF, Washietl S, Hofacker IL: miRNAMap: genomic maps of microRNA genes and their target genes in mammalian genomes. Nucleic Acids Res 2006:D I35-DI39.

6I. Kent WJ: BLAT: the BLAST-like alignment tool. Genome Res 2002, I 2:656-664.

62. Hayashita $\mathrm{Y}$, Osada H, Tatematsu $\mathrm{Y}$, Yamada H, Yanagisawa $\mathrm{K}$, Tomida S, Yatabe Y, Kawahara K, Sekido Y, Takahashi T: A polycistronic microRNA cluster, miR-17-92, is overexpressed in human lung cancers and enhances cell proliferation. Cancer Res 2005, 65:9628-9632.

63. UCSC MULTIZ alignments [http://hgdownload.cse.ucsc.edu/ goldenPath/hg|8/multiz|7way/]

64. Blanchette M, Kent WJ, Riemer C, Elnitski L, Smit AF, Roskin KM, Baertsch R, Rosenbloom K, Clawson H, Green ED, et al:: Aligning multiple genomic sequences with the threaded blockset aligner. Genome Res 2004, 14:708-7I5.

65. Lander ES, Linton LM, Birren B, Nusbaum C, Zody MC, Baldwin J, Devon K, Dewar K, Doyle M, FitzHugh W, et al.: Initial sequencing and analysis of the human genome. Nature 200I, 409:860-92I.

66. Consortium CSaA: Initial sequence of the chimpanzee genome and comparison with the human genome. Nature 2005, 437:69-87.

67. Waterston RH, Lindblad-Toh K, Birney E, Rogers J, Abril JF, Agarwal P, Agarwala R, Ainscough R, Alexandersson M, An P, et al.: Initial sequencing and comparative analysis of the mouse genome. Nature 2002, 420:520-562.

68. Gibbs RA, Weinstock GM, Metzker ML, Muzny DM, Sodergren EJ, Scherer S, Scott G, Steffen D, Worley KC, Burch PE, et al.: Genome sequence of the Brown Norway rat yields insights into mammalian evolution. Nature 2004, 428:493-52I.

69. Hillier LW, Miller W, Birney E, Warren W, Hardison RC, Ponting CP Bork P, Burt DW, Groenen MA, Delany ME, et al.: Sequence and comparative analysis of the chicken genome provide unique perspectives on vertebrate evolution. Nature 2004, 432:695-7।6.

70. Aparicio S, Chapman J, Stupka E, Putnam N, Chia JM, Dehal P, Christoffels A, Rash S, Hoon S, Smit A, et al.: Whole-genome shotgun assembly and analysis of the genome of Fugu rubripes. Science 2002, 297:| $|30|-|3| 0$.

7I. Jaillon O, Aury JM, Brunet F, Petit JL, Stange-Thomann N, Mauceli E, Bouneau L, Fischer C, Ozouf-Costaz C, Bernot A, et al:: Genome duplication in the teleost fish Tetraodon nigroviridis reveals the early vertebrate proto-karyotype. Nature 2004, 43 I:946-957.

72. PhyloGif Program for Phylogenetic Trees [http:// genome.ucsc.edu/cgi-bin/phyloGif]

73. Margulies EH, Maduro VV, Thomas PJ, Tomkins JP, Amemiya CT, Luo $M$, Green ED: Comparative sequencing provides insights about the structure and conservation of marsupial and monotreme genomes. Proc Natl Acad Sci USA 2005, 102:3354-3359.

74. Benos laboratory web server. [http://www.benoslab.pitt.edu]

75. Loots GG, Ovcharenko I, Pachter L, Dubchak I, Rubin EM: rVista for comparative sequence-based discovery of functional transcription factor binding sites. Genome Res 2002, I 2:832-839. 